\title{
Proton quantum coherence observed in water confined in silica nanopores
}

\author{
V. Garbuio and C. Andreani \\ Dipartimento di Fisica, Università degli Studi di Roma “Tor Vergata," Via della Ricerca Scientifica 1, \\ 00133 Roma, Italy and Centro NAST (Nanoscienze \& Nanotecnologie \& Strumentazione), Università degli \\ Studi di Roma "Tor Vergata," Via della Ricerca Scientifica 1, 00133 Roma, Italy \\ S. Imberti $^{\mathrm{a})}$ \\ Centro NAST (Nanoscienze \& Nanotecnologie \& Strumentazione), Università degli Studi di Roma "Tor \\ Vergata,”Via della Ricerca Scientifica 1, 00133 Roma, Italy
}

\begin{abstract}
A. Pietropaolo
Dipartimento di Fisica, Università degli Studi di Roma "Tor Vergata," Via della Ricerca Scientifica 1, 00133 Roma, Italy and Centro NAST (Nanoscienze \& Nanotecnologie \& Strumentazione), Università degli Studi di Roma "Tor Vergata," Via della Ricerca Scientifica 1, 00133 Roma, Italy

G. F. Reiter

Physics Department, University of Houston, 4800 Calhoun Road, Houston, Texas 77204, USA

R. Senesi ${ }^{\text {b) }}$

Dipartimento di Fisica, Università degli Studi di Roma “Tor Vergata," Via della Ricerca Scientifica 1, 00133 Roma, Italy and Centro NAST (Nanoscienze \& Nanotecnologie \& Strumentazione), Università degli Studi di Roma "Tor Vergata," Via della Ricerca Scientifica 1, 00133 Roma, Italy

M. A. Ricci

Dipartimento di Fisica E. Amaldi, Università degli Studi Roma TRE, Via della Vasca Navale 84, 00146 Roma, Italy
\end{abstract}

(Received 3 July 2007; accepted 31 August 2007; published online 15 October 2007)

Deep inelastic neutron scattering measurements of water confined in nanoporous xerogel powders, with average pore diameters of 24 and $82 \AA$, have been carried out for pore fillings ranging from $76 \%$ to nearly full coverage. DINS measurements provide direct information on the momentum distribution $n(p)$ of protons, probing the local structure of the molecular system. The observed scattering is interpreted within the framework of the impulse approximation and the longitudinal momentum distribution determined using a model independent approach. The results show that the proton momentum distribution is highly non-Gaussian. A bimodal distribution appears in the $24 \AA$ pore, indicating coherent motion of the proton over distances $d$ of approximately $0.3 \AA$. The proton mean kinetic energy $\left\langle E_{K}\right\rangle_{W}$ of the confined water molecule is determined from the second moment of $n(p)$. The $\left\langle E_{K}\right\rangle_{W}$ values, higher than in bulk water, are ascribed to changes of the proton dynamics induced by the interaction between interfacial water and the confining surface. (C) 2007 American Institute of Physics. [DOI: 10.1063/1.2789436]

\section{INTRODUCTION}

Liquid water is a topic which continues to attract intense interest and motivate a large number of experimental and theoretical investigations. ${ }^{1-8}$ Most recently, great attention has been devoted to the study of water confined in nanoporous systems of different geometries, in both solid or gel phases, or in proximity of macromolecules and surfaces, because of its biological and technological importance. ${ }^{9-20}$ These studies are very relevant for a deep understanding of a large variety of processes ranging from catalysis and soil chemistry to biological processes, such as protein folding or ionic transport in membranes. ${ }^{9}$ It is well assessed that microscopic properties of water molecules interacting with the confining surfaces differ from those in the bulk phase. The

${ }^{a)}$ Present address: ISIS Facility, Rutherford Appleton Laboratory, Chilton, Didcot, Oxon OX11 OQX, United Kingdom.

${ }^{b)}$ Electronic mail: roberto.senesi@ roma2.infn.it competition between water-water and water-confining medium interactions leads to the appearance of new interesting physical properties. For instance, crystallization is suppressed at least for the first two layers and confined water can thus be supercooled easily and freezes forming a glass below $180 \mathrm{~K} .^{10,11}$ In addition, changes in both structural and dynamical properties of water occur. These changes are introduced both by the confining geometry and by the enhanced surface interaction due to the large surface to volume ratio of porous materials and/or due to an interruption of the bulk correlation length. Because water molecules interact with the wall particles, these changes may depend on whether the nature of these interactions is hydrophilic or hydrophobic. Up to now, however, there is no general theory predicting the behavior of confined liquids and solids or the relative importance of surface interaction versus confinement.

Several structural and dynamical studies of water in con- 
fined geometries have been performed using different and complementary experimental techniques. These include $\mathrm{x}$-ray ${ }^{12}$ and neutron diffraction measurements ${ }^{13}$ and dynamical investigation such as diffusive relaxation and vibrational properties of water studied by incoherent quasielastic ${ }^{14,15}$ and inelastic neutron scattering, ${ }^{17,21-23} \mathrm{NMR}^{24}{ }^{27}$ optical spectroscopy, ${ }^{25,26}$ and viscosity measurements. ${ }^{27,28}$ These studies reveal that confinement induces changes in thermodynamics, phase behavior, and dynamics of water, which depend on the nature and morphology of the confining surfaces. It appears that the interaction of water molecules with charged and polar surfaces may induce patch breaking in the hydrogen bond network, while nonpolar interactions enhance the formation of short range order. ${ }^{29}$ NMR and quasielastic neutron scattering experiments show a slowing down of water dynamics near a hydrophilic surface as compared to bulk water. ${ }^{17,21,30}$ Several computer simulations have been also performed on water confined in different nanoporous materials. ${ }^{31-38}$ These indicate that the diffusion dynamics of water, in the picosecond time scale, typically slows down near a nonattractive rough surface and speeds up near a nonattractive smooth surface. ${ }^{39}$ Once confined in Vycor nanopores, ${ }^{40}$ for example, water molecules exhibit at least two distinct regimes: a slow diffusion near the surface and a fast dynamics far away from the surface. ${ }^{14,31,33,41-45}$

This paper reports a study of the short-time proton dynamics in water confined in silica nanopores (xerogel powder), via deep inelastic neutron scattering (DINS) measurements, using the VESUVIO spectrometer at the ISIS spallation neutron source. ${ }^{46}$ DINS measurements are performed in the attosecond time scale (i.e., $10^{-15}-10^{-16} \mathrm{~s}$ ). These measurements provide unique information on the proton momentum distribution $n(p)$ and proton mean kinetic energy $\left\langle E_{K}\right\rangle_{W}$ of the confined water molecules, physical quantities which are influenced by the local environment of the atoms. ${ }^{47}$ The aim of the present experiments is to study the effect on proton dynamics due to the confinement in silica pores with different pore diameters. In previous DINS studies, performed on $\mathrm{H}_{2} \mathrm{~S}$ and supercritical $\mathrm{H}_{2} \mathrm{O},{ }^{5,48}$ it has been shown that DINS data can be interpreted with a proton momentum distribution calculated by performing an orientational average of an anisotropic Gaussian function, which reflects the molecular geometry. In the present study, a slightly different approach has been followed. The experimental response function $F(y)$ has been reproduced using a model independent non-Gaussian lineshape ${ }^{47}$ successfully employed in previous work on ice, bulk, and confined water. ${ }^{6}$ In Section II, the DINS theory is reviewed. Section III illustrates the experiments and data analysis. In Section IV, results are presented and discussed. Conclusions are reported in Sec. V.

\section{DINS THEORY}

In the limit of high wavevector transfer $q$, the scattering process of the individual protons in water resembles closely the scattering of freely moving particles. In this limit, the dynamical structure factor $S(\mathbf{q}, \omega)$ can be expressed assuming that a single particle of the system is struck by the scat- tering probe and that this particle recoils freely from the collision, with interparticle interaction in the final state being negligible. The fraction of neutrons scattered into a given angle with a given energy depends only on the probability that the proton had a particular momentum $p$ at the time it was struck by the neutron (i.e., in the initial state). The theoretical basis of DINS is the impulse approximation (IA). The validity of this approximation in neutron scattering has been discussed by many authors in previous papers. ${ }^{49,50}$ The IA effectively treats the scattering event as single atom "billiard ball" scattering, with conservation of momentum and kinetic energy of neutron plus target atom. The dynamic structure factor for the proton is expressed as

$$
S(\mathbf{q}, \omega)=\hbar \int n(\mathbf{p}) \delta\left[\hbar \omega-\hbar \omega_{r}-\frac{\mathbf{p} \cdot \hbar \mathbf{q}}{M}\right] d \mathbf{p},
$$

where $M$ is the hydrogen mass, $n(\mathbf{p})$ the proton momentum distribution, and $\hbar \omega_{r}$ the recoil energy:

$$
\hbar \omega_{r}=\frac{\hbar^{2} q^{2}}{2 M} .
$$

It has to be stressed that in a molecular system, when the energy scales corresponding to intra- and intermolecular motions are sufficiently well separated, two distinct regimes can be envisaged for the applicability of IA. ${ }^{51}$ In the first one, the energy transfer is large compared to the typical energy for collective excitations of the system but lower than the minimum internal excitation energy of a single molecule. This regime, where one can study the rototranslational dynamics of the molecule as a whole, is accessible through conventional inelastic neutron scattering experiments, in a range of $q$ where the response is incoherent. A second regime, where the energy transfer greatly exceeds the energy scale of internal excitations of the molecules, allows the investigation of the dynamics of the atoms within the molecule and is accessible via DINS technique. This is the regime of application of Eq. (2.1).

Within the IA framework, one assumes an infinite value for the wavevector $(q \rightarrow \infty)$ and introduces a scaling West variable $y$, where the variables for energy $(\hbar \omega)$ and momentum $(\hbar q)$ transfers are coupled through the relation

$$
y=\frac{M}{\hbar q}\left(\omega-\frac{\hbar q^{2}}{2 M}\right) .
$$

In an isotropic system a scaling response function is introduced, $J(y) .{ }^{52}$ This function can be expressed in terms of the longitudinal momentum distribution of the single proton $n(\mathbf{p})$ :

$$
J(y, \hat{\mathbf{q}})=\int d \mathbf{p} n(\mathbf{p}) \delta\left(y-\frac{\mathbf{p} \cdot \hat{\mathbf{q}}}{\hbar}\right) .
$$

For the atom of mass $M$, the $J(y, \hat{\mathbf{q}})$ function is formally the Radon transform of the momentum distribution and represents the probability distribution of the momentum $\mathbf{p}$ along the direction of q. This is analogous to the Compton profile, measured in Compton scattering of photons from electrons. Although the Radon transform of $n(\mathbf{p})$ for a particular $y$ and $\hat{\mathbf{q}}$ is an average over a plane, no information is lost, and $n(\mathbf{p})$ 
can be reconstructed from $J(y, \hat{\mathbf{q}})$. In the isotropic case, from Eq. (2.4) one obtains

$$
n(p)=-\left.\frac{1}{2 \pi \hbar^{3} y} \frac{d}{d y} J(y)\right|_{\hbar y=p},
$$

and from Eqs. (2.1) and (2.4) one can also write

$$
S(q, \omega)=\frac{M}{\hbar q} J(y) .
$$

The single particle mean kinetic energy $\left\langle E_{K}\right\rangle$ is related to the second moment of the response function $J(y)$ via

$$
\left\langle E_{K}\right\rangle=\frac{3 \hbar^{2}}{2 M} \int_{-\infty}^{\infty} y^{2} J(y) d y=\frac{3 \hbar^{2}}{2 M} \sigma^{2} .
$$

A non-Gaussian model for $J(y)$ can be considered ${ }^{49}$ This uses a general expansion of the response function in Hermite polynomials $H_{n}(x)$, the Gram-Charlier series. ${ }^{47}$ In the case of a liquid, where the average wavevector distribution has no angular dependence, the general expression can be written in the form

$$
J(y)=\frac{e^{-y^{2} / 2 \sigma^{2}}}{\sqrt{2 \pi \sigma^{2}}} \sum_{n} \frac{a_{n}}{2^{2 n} n !} H_{2 n}\left(\frac{y}{\sqrt{2} \sigma}\right) .
$$

For finite values of the wavevector, the response function in Eq. (2.4) retains the $q$ dependence and Eq. (2.6) becomes:

$$
S(q, \omega)=\frac{M}{\hbar q} J(y, q) .
$$

Deviations from the IA give rise to final state effects. ${ }^{53}$ These can be properly accounted for in a non-Gaussian model [Eq. (2.8)] by the addition of further terms proportional to $1 / q\left[H_{3}(y / \sqrt{2 \sigma})\right]$ [see Eq. (4.1)].

\section{EXPERIMENT AND DATA ANALYSIS}

Two kinds of xerogel powders have been synthesized with average pore diameters $d$ of 24 and $82 \AA$, respectively. The materials were obtained by porous glasses originally produced via the Sol-Gel method, ${ }^{54,55}$ formed by mixing liquid alkoxide precursors, tetramethylortho-silicate (TMOS), and tetraethoxysilane (TEOS), with different solvents, water, and ethanol. Specific catalysts and control chemicals were added in order to improve the process. Final solutions were then stirred and stored at room temperature for two months, in order to complete the polymerization process. The xerogel powders have been characterized (see Table I) in terms of both pore size distribution functions and specific surface area, via Brunauer, Emmett, Teller (BET) method, measuring adsorption/desorption method, measuring adsorption/ desorption isotherms. ${ }^{56}$ The powder samples had an approximate volume of $30 \mathrm{~cm}^{3}$ and were vacuum pumped in situ before the measurements for $12 \mathrm{~h}$ at $70^{\circ} \mathrm{C}$. For each pore size, water was adsorbed in dry silica xerogel powder (about $15 \mathrm{~cm}^{3}$ in volume) by a hydration protocol in a controlled environment. The hydration procedure made use of three Petri dishes filled with dry xerogel powder and a fourth Petri dish containing a saturated solution of potassium nitrate
TABLE I. Physical parameters of the two xerogel samples used in the experiments, as derived from BET analysis (Ref. 56). The hydration level is defined as the ratio between water and dry xerogel weights.

\begin{tabular}{lccccc}
\hline \hline & $\begin{array}{c}\text { Average } \\
\text { pore } \\
\text { size } \\
(\AA)\end{array}$ & $\begin{array}{c}\text { Total } \\
\text { pore } \\
\text { volume } \\
\left(\mathrm{cm}^{3} / \mathrm{g}\right)\end{array}$ & $\begin{array}{c}\text { Specific } \\
\text { surface } \\
\text { area } \\
\left(\mathrm{m}^{2} / \mathrm{g}\right)\end{array}$ & $\begin{array}{c}\text { Hydration } \\
\text { level } \\
(\%)\end{array}$ & $\begin{array}{c}\text { Pore } \\
\text { filling } \\
(\%)\end{array}$ \\
\hline $\begin{array}{l}\mathrm{TEOS}- \\
\mathrm{Si}\left(\mathrm{OC}_{2} \mathrm{H}_{5}\right)_{4}\end{array}$ & 24 & 0.348 & 727 & 26.4 & 76 \\
$\mathrm{TMOS}_{4}$ & 82 & 1.10 & 500 & 108 & 98 \\
$\mathrm{Si}\left(\mathrm{OCH}_{3}\right)_{4}$ & & & & & \\
\hline \hline
\end{tabular}

$\left(\mathrm{KNO}_{3}\right)$ and water; all dishes were introduced in desiccators to ensure an insulated environment during all the hydration process time. The sample was maintained in this controlled environment for about $96 \mathrm{~h}$. The level of hydration was determined by weight measurements. Once the equilibrium between the xerogel and the solution was reached, the xerogel with pore sizes of 24 and $82 \AA$ were hydrated at water contents of $26.4 \%$ and $108 \%$ of the dry weight, respectively. Due to the hygroscopic properties of both samples, one can assume that water is completely adsorbed in the pores of the material and calculate the corresponding pore filling values: $76 \%$ and $98 \%$ for the xerogel powders with average pore sizes of 24 and $82 \AA$, respectively (see Table I). These values have been derived assuming the water molecule to be a sphere with diameter of $3.16 \AA$.

The experiments have been performed at the ISIS spallation neutron source (Rutherford Appleton Laboratory, Chilton, Didcot, UK) using the inverse geometry spectrometer VESUVIO. $^{46}$ The incident neutron energy range, $10^{-3}-10^{4} \mathrm{eV}$, makes VESUVIO a unique instrument for DINS. ${ }^{57}$ The spectrometer is equipped with forward and backscattering detector banks, specifically designed for measurements of single particle proton dynamics in the wavevector and energy transfer ranges $20 \AA^{-1}<q<250 \AA^{-1}$ and $1 \mathrm{eV}<\hbar \omega<20 \mathrm{eV}$, respectively. ${ }^{47}$ Scattered neutrons were recorded by ${ }^{6} \mathrm{Li}$ glass scintillators, located at a distance of about $0.5 \mathrm{~m}$ from the sample position, in the angular range $30^{\circ}<2 \theta<70^{\circ}$. The kinematics of the scattering events is reconstructed via the standard time of flight (tof) technique. ${ }^{46}$

For each scattering angle the final energy of the scattered neutrons, $E_{1}$, is selected using the resonance filter configuration, ${ }^{58,59}$ using a thin (about $10 \mu \mathrm{m}$ thickness) filter of gold, ${ }^{197} \mathrm{Au}$, which absorbs neutrons in a narrow range of energies. For each fixed angle detector (denoted by the index $n$ ), the main component of the instrumental resolution function, $R_{n}(y, q)$, is determined from the absorption lineshape of the filter. ${ }^{60}$ This function is approximately described by the convolution of a Lorentzian shape, originating in the BreitWigner form for the nuclear resonance, and a Gaussian shape due to the Doppler broadening associated with atomic vibrations in the filter material. ${ }^{58}$ In the case of gold filters the intrinsic Breit-Wigner component of the resonance dominates the Gaussian component, with a final energy $E_{1}=4908 \mathrm{meV}$ with half width at half maximum (HWHM) $\Delta E_{1} \simeq 140 \mathrm{meV}$. Uncertainties in the geometrical instrument parameters, such as flight paths, angles, etc., ${ }^{60}$ contribute to 
TABLE II. Values of some of the scattering angles used in the DINS measurements of water confined in xerogel powders and of wavevector transfer $\bar{q}$ (in $\AA^{-1}$ ) corresponding, for each angle, to the maximum of the recoil peak in $F_{n}^{\exp }(y, 2 \theta)$ function (see Fig. 2). Parameters describing the resolution function $R_{n}(y, 2 \theta)$ on VESUVIO, for mass $M=1.0079$ a.m.u. and ${ }^{197} \mathrm{Au}$ resonance foil, as a function of different scattering angles are also reported: $\sigma_{G}$ indicates the standard deviation of the whole Gaussian component (energy plus geometrical contributions); $\Gamma_{E} / 2$ is the half width at half maximum (HWHM) of the Lorentzian energy contribution.

\begin{tabular}{|c|c|c|c|c|c|c|c|}
\hline \multicolumn{4}{|c|}{ Xerogel, $24 \AA$ pore size } & \multicolumn{4}{|c|}{ Xerogel, $82 \AA$ pore size } \\
\hline $\begin{array}{l}2 \theta \\
(\operatorname{deg})\end{array}$ & $\begin{array}{c}\bar{q} \\
\left(\AA^{-1}\right)\end{array}$ & $\begin{array}{c}\sigma_{G} \\
\left(\AA^{-1}\right)\end{array}$ & $\begin{array}{l}\Gamma_{E} / 2 \\
\left(\AA^{-1}\right)\end{array}$ & $\begin{array}{c}2 \theta \\
(\mathrm{deg})\end{array}$ & $\begin{array}{c}\bar{q} \\
\left(\AA^{-1}\right)\end{array}$ & $\begin{array}{c}\sigma_{G} \\
\left(\AA^{-1}\right)\end{array}$ & $\begin{array}{l}\Gamma_{E} / 2 \\
\left(\AA^{-1}\right)\end{array}$ \\
\hline 32.1 & 30.6 & 0.71 & 1.24 & 32.5 & 31.2 & 0.67 & 0.74 \\
\hline 34.5 & 33.5 & 0.70 & 1.14 & 34.4 & 33.4 & 0.67 & 0.69 \\
\hline 36.6 & 36.3 & 0.69 & 1.06 & 36.4 & 36.0 & 0.68 & 0.64 \\
\hline 38.7 & 39.1 & 0.70 & 1.00 & 38.2 & 38.7 & 0.67 & 0.60 \\
\hline 40.7 & 42.0 & 0.68 & 0.92 & 40.4 & 41.9 & 0.66 & 0.56 \\
\hline 43.0 & 45.4 & 0.68 & 0.86 & 42.5 & 45.0 & 0.66 & 0.52 \\
\hline 45.0 & 48.8 & 0.67 & 0.80 & 44.7 & 48.4 & 0.66 & 0.49 \\
\hline 47.2 & 52.7 & 0.67 & 0.74 & 46.7 & 52.2 & 0.65 & 0.45 \\
\hline 51.5 & 61.4 & 0.66 & 0.64 & 51.2 & 61.6 & 0.65 & 0.39 \\
\hline 52.9 & 64.7 & 0.66 & 0.61 & 53.4 & 66.5 & 0.66 & 0.36 \\
\hline 54.7 & 69.1 & 0.66 & 0.57 & 55.3 & 71.9 & 0.66 & 0.34 \\
\hline 56.8 & 74.6 & 0.66 & 0.53 & 57.5 & 77.5 & 0.66 & 0.31 \\
\hline 59.2 & 81.9 & 0.65 & 0.49 & 59.6 & 84.3 & 0.66 & 0.29 \\
\hline 61.2 & 89.1 & 0.65 & 0.45 & 61.6 & 91.9 & 0.66 & 0.27 \\
\hline 63.3 & 97.5 & 0.64 & 0.42 & 63.8 & 100.4 & 0.65 & 0.25 \\
\hline 65.6 & 108.1 & 0.64 & 0.38 & 65.7 & 110.1 & 0.65 & 0.23 \\
\hline
\end{tabular}

the angular dependent resolution function lineshapes producing a further Gaussian broadening. In the present experiment, the Lorentzian and the Gaussian components of the resolution functions are reported in Table II. ${ }^{59,61,62}$ It can be appreciated that both Gaussian and Lorentzian contributions to the $R_{n}(y, q)$ function decrease as $q$ increases, i.e., scattering angle increases.

DINS measurements have been performed on hydrated and dry xerogel samples, for both pore sizes, 24 and $82 \AA$, at $T=293 \mathrm{~K}$. All samples were contained in square flat aluminum cell $6 \mathrm{~cm}$ width, $6 \mathrm{~cm}$ height, and $0.1 \mathrm{~cm}$ internal thickness. Experimental DINS data have been analyzed using the standard VESUVIO routines. ${ }^{63}$ Examples of normalized tof spectra are shown in Fig. 1, for three selected values of the scattering angle. At each angle, two distinct peaks are visible: the first one, at smaller tof, is the DINS recoil peak associated with hydrogen atoms in the sample: hydrogen atoms in water molecules-within the pore and close to the pore surface - and hydrogen atoms within the xerogel matrices, due to the presence of silanol $(\mathrm{SiOH})$ groups. ${ }^{64}$ In order to derive a reliable lineshape for the response function of the proton in water, contributions from different hydrogen components need to be evaluated (see Sec. IV). The second peak in Fig. 1, centered at about $370 \mu \mathrm{s}$, is associated with the scattering of all other atoms with higher masses in the sample (silicon, oxygen, and aluminum). It is also evident from this figure that the two peaks tend to be closer at smaller scattering angles. The small sample thickness guarantees a small contribution from multiple scattering signal. The latter was evaluated by a Monte Carlo simulation ${ }^{62}$ and then subtracted from all the spectra. Results of this simulation are shown in the inset of Fig. 1. In order to obtain a signal with better statistics, data from the $24 \AA$ pore size xerogel, recorded at scattering angles within $2^{\circ}$, have been summed together. A further step of the data reduction consists in transforming tof spectra in $y$ space. Within the convolution approximation ${ }^{47}$ the experimental response function for individual detectors, $F_{n}^{\exp }(y, 2 \theta)$, can be expressed as a convolution of the proton response function, $J(y, 2 \theta)$, and the resolution function, $R_{n}(y, 2 \theta)$ :

$$
F_{n}^{\exp }(y, 2 \theta)=J(y, 2 \theta) \otimes R_{n}(y, 2 \theta) .
$$

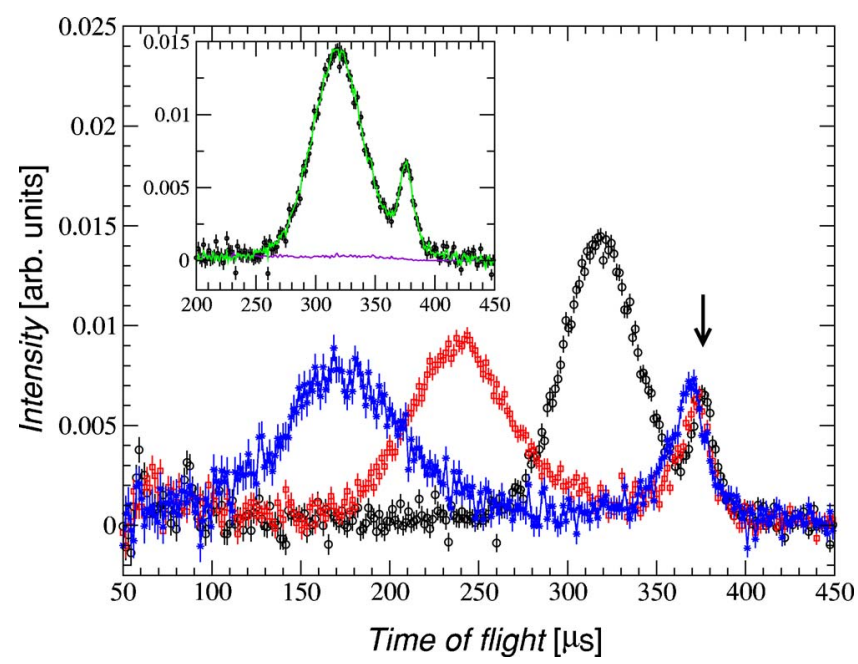

FIG. 1. (Color online) Time-of-flight DINS spectra from water confined in a xerogel powder with average pore size of $24 \AA$ for three scattering angles: $34.5^{\circ}$ (circles), $52.9^{\circ}$ (squares), and $65.6^{\circ}$ (stars). By increasing scattering angle, peak associated with recoil from all hydrogen atoms (at higher tof) moves apart from recoil peak, associated with all the other elements ( $\mathrm{Si}, \mathrm{O}$, $\mathrm{Al}$ ). In the inset, the results of the Monte Carlo simulation are shown together with the experimental spectrum for the scattering angle $34.5^{\circ}$ (black circles): the green line is the simulated total scattering, and the violet line is the multiple scattering contribution. 


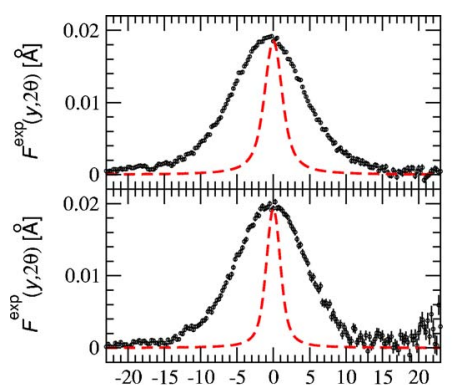

(a)

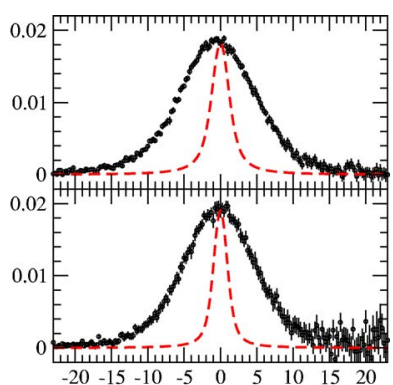

(b)

$$
y\left[\AA^{-1}\right]
$$

FIG. 2. (Color online) Experimental response functions $F_{n}^{\exp }(y, 2 \theta)$ (with error bars) for water confined in xerogel and corresponding resolution functions (dashed lines); (a) xerogel pore size $24 \AA$ for two scattering angles: $38.4^{\circ}$ (top panel) and $52.4^{\circ}$ (bottom panel); (b) xerogel pore size $82 \AA$ for two scattering angles: $38.2^{\circ}$ (top panel) and $52.7^{\circ}$ (bottom panel).

Examples of $F_{n}^{\exp }(y, 2 \theta)$ spectra, as a function of $y$, at different scattering angles, are shown in Fig. 2, together with the corresponding resolution functions. From this figure one can notice that, by increasing scattering angle (from top to bottom), the peak in $F_{n}^{\exp }(y, 2 \theta)$ moves toward a value centered at $y=0$, as expected within the impulse approximation. $^{47}$

In order to interpret data in terms of the dynamical structure factor and neutron Compton profile of Eq. (2.9) where $q$ is fixed, it is useful to express experimental spectra also in terms of constant wavevector transfer $q$. This goal has been achieved by converting constant angle spectra to constant wavevector ones through the following procedure. The experimental tof spectra, neutron counts versus time of flight, have been transformed to neutron counts versus energy transfer at constant scattering angle, $S(2 \theta, \omega)$, using standard routines ${ }^{47}$ which take into account, e.g., the incident neutron spectrum, the energy-dependent monitor efficiency, and energy-time Jacobian; in this conversion, for each time of flight bin, the corresponding values of wavevector and energy transfer are calculated. In order to convert these data in $S(q, \omega)$, a procedure has been implemented to perform a rebinning of data taken at constant scattering angle in the $(q, \omega)$ plane onto a rectangular $(q, \omega)$ grid. The constant $q$ width onto which the data have to be rebinned is chosen to have a width in $q$ which is larger than the $q$ uncertainty for each detector. A typical $q$ uncertainty for the present measurements is of the order of $1.5 \AA^{-1}{ }^{65}$ Further details of this procedure and a comparison with other methods for obtaining equivalent constant- $q$ scans with fixed-angle spectra ${ }^{66}$
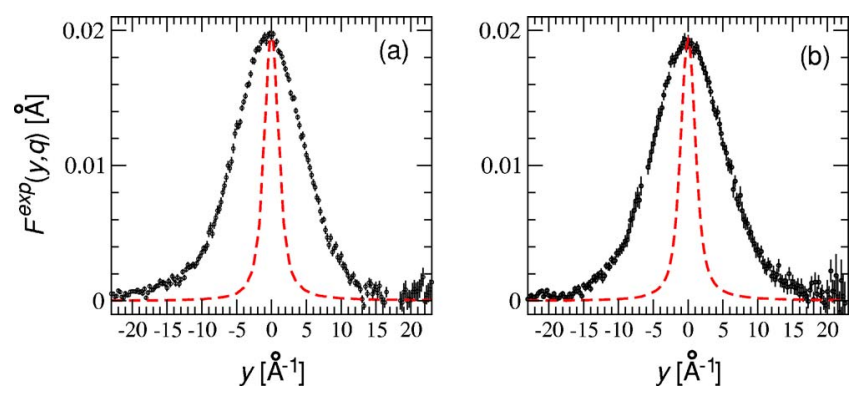

FIG. 3. (Color online) Experimental response functions $F^{\exp }(y, q)$ (with error bars) and corresponding resolution functions (dashed lines) for water confined in xerogel with average pore diameters $d$ (a) $24 \AA$ and (b) $82 \AA$ at constant momentum transfers $q=53 \pm 3 \AA^{-1}$ and $q=50 \pm 3 \AA^{-1}$, respectively.

will be matter of a separate paper. The constant angle to constant $q$ conversion is therefore unique and depends on the width chosen for the $q$ bins, and involves the application of the Jacobian factor: ${ }^{67}$

$$
S(q, \omega)=S(2 \theta, \omega)\left|\frac{\partial(2 \theta)}{\partial q}\right| .
$$

The final step consisted in obtaining the experimental Compton profiles at constant $q$, applying Eq. (2.9) and obtaining the data sets $F^{\exp }(y, q)$. In particular, a set of $F^{\exp }(y, q)$ spectra in $y$ space has been obtained for the following values (and $q$ width) of wavevector transfer: $38 \pm 2,45 \pm 2,53 \pm 3$, $70 \pm 5 \AA^{-1}$. The constant $q$ data sets have then been rebinned into $y$ bins, weighted by their errors. ${ }^{68}$ For these constant wavevector transfer data, the corresponding constant $q$ resolutions have been obtained through an identical procedure applied to the tof resolution functions evaluated by a Monte Carlo simulation, ${ }^{62,69,70} R_{n}$ (tof, $\left.2 \theta\right)$. Examples of $F^{\exp }(y, q)$ spectra at constant $q$ are shown in Fig. 3 together with the corresponding resolutions, $R(y, q)$.

Within the incoherent approximation, one can express the experimental response function for the individual detector as a sum of contributions of the different kinds of hydrogen atoms in the sample:

$$
\begin{aligned}
F_{n}^{\exp }(y, 2 \theta)= & {\left[\alpha_{1} J_{H_{1}}(y, 2 \theta)+\alpha_{2} J_{H_{2}}(y, 2 \theta)+\cdots\right] } \\
& \otimes R_{n}(y, 2 \theta),
\end{aligned}
$$

where $\alpha_{1}$ and $\alpha_{2}$ are the relative fractions associated with different hydrogen atoms.

TABLE III. Results obtained from individual fits of $F_{n}^{\exp }(y, 2 \theta)$ data sets for the hydrated xerogel samples with

\begin{tabular}{|c|c|c|c|c|c|c|c|}
\hline \multicolumn{4}{|c|}{ Xerogel $_{H}, 24 \AA$} & \multicolumn{4}{|c|}{ Xerogel $_{H}, 82 \AA$} \\
\hline $\begin{array}{c}2 \theta \\
(\operatorname{deg})\end{array}$ & $\begin{array}{c}\sigma_{H} \\
\left(\AA^{-1}\right)\end{array}$ & $\begin{array}{c}c_{4 H} \\
\left(\times 10^{-2}\right)\end{array}$ & $\begin{array}{l}\left\langle E_{K}\right\rangle_{H} \\
(\mathrm{meV})\end{array}$ & $\begin{array}{c}2 \theta \\
(\operatorname{deg})\end{array}$ & $\begin{array}{c}\sigma_{H} \\
\left(\AA^{-1}\right)\end{array}$ & $\begin{array}{c}c_{4 H} \\
\left(\times 10^{-2}\right)\end{array}$ & $\begin{array}{l}\left\langle E_{K}\right\rangle_{H} \\
(\mathrm{meV})\end{array}$ \\
\hline 38.2 & $6.0 \pm 0.1$ & $2.5 \pm 0.2$ & $225 \pm 7$ & 37.5 & $5.4 \pm 0.1$ & $1.6 \pm 0.2$ & $186 \pm 6$ \\
\hline 59.2 & $6.0 \pm 0.1$ & $2.7 \pm 0.1$ & $225 \pm 7$ & 58.9 & $5.5 \pm 0.2$ & $1.7 \pm 0.3$ & $188 \pm 11$ \\
\hline 64.5 & $5.9 \pm 0.1$ & $2.7 \pm 0.2$ & $215 \pm 6$ & 64.9 & $5.7 \pm 0.2$ & $1.9 \pm 0.4$ & $190 \pm 14$ \\
\hline
\end{tabular}
average pore sizes of 24 and $82 \AA$, at three selected scattering angles. The suffix $H$ refers to hydrated xerogel sample: $\sigma_{H}$ and $\left\langle E_{K}\right\rangle_{H}$ represent standard deviation and mean kinetic energy of the whole (silanol and water) hydrogen atoms. The $c_{4 H}$ is the non-Gaussian coefficient resulting from the fits. 
TABLE IV. Results obtained from simultaneous fit of $F_{n}^{\exp }(y, 2 \theta)$ data for both dry xerogel and hydrated xerogel samples, for pore sizes of 24 and $82 \AA$. The suffixes $D$ and $H$ refer to dry and hydrated xerogel samples: $\sigma_{D}, \sigma_{H}$ and $\left\langle E_{K}\right\rangle_{D},\left\langle E_{K}\right\rangle_{H}$ represent standard deviations and mean kinetic energies of dry and hydrated samples. The $c_{4 D}$ and $c_{4 H}$ are the non-Gaussian coefficients, resulting from the fits.

\begin{tabular}{|c|c|c|c|c|c|c|}
\hline \multirow[b]{2}{*}{$\begin{array}{c}d \\
(\AA)\end{array}$} & \multicolumn{3}{|c|}{ Xerogel $_{D}$} & \multicolumn{3}{|c|}{ Xerogel $_{H}$} \\
\hline & $\begin{array}{c}\sigma_{D} \\
\left(\AA^{-1}\right)\end{array}$ & $\begin{array}{c}c_{4 D} \\
\left(\times 10^{-2}\right)\end{array}$ & $\begin{array}{l}\left\langle E_{K}\right\rangle_{D} \\
(\mathrm{meV})\end{array}$ & $\begin{array}{c}\sigma_{H} \\
\left(\AA^{-1}\right)\end{array}$ & $\begin{array}{c}c_{4 H} \\
\left(\times 10^{-2}\right)\end{array}$ & $\begin{array}{l}\left\langle E_{K}\right\rangle_{H} \\
(\mathrm{meV})\end{array}$ \\
\hline 24 & $6.90 \pm 0.05$ & $2.87 \pm 0.08$ & $296 \pm 5$ & $5.95 \pm 0.03$ & $2.81 \pm 0.05$ & $221 \pm 2$ \\
\hline 82 & $6.60 \pm 0.08$ & $2.76 \pm 0.13$ & $271 \pm 7$ & $5.47 \pm 0.02$ & $1.56 \pm 0.07$ & $186 \pm 1$ \\
\hline
\end{tabular}

\section{RESULTS AND DISCUSSION}

In order to derive the proton momentum distribution and mean kinetic energy of water, a three step analysis has been performed. At first, the contribution due to silanol-hydrogen atoms have been evaluated using dry xerogel data sets (hereafter indicated with suffix $D$ ). The second step consisted in calculating the contributions from both water-hydrogen atoms and silanol-hydrogen atoms using the hydrated xerogel data sets (hereafter indicated with suffix $H$ ). The percentage of silanol-hydrogen atoms with respect to the total number of hydrogen atoms has then been estimated as explained in the following. The individual spectra for dry and hydrated xerogel data sets, at both constant $q\left[F^{\exp }(y, q)\right]$ and angle $\left[F_{n}^{\exp }(y, 2 \theta)\right]$, have been fitted using Eq. (2.8), already successfully employed for liquid $\mathrm{H}_{2} \mathrm{O}$ (Refs. 6 and 47) and for ice. ${ }^{6}$ Indeed previous DINS experiments on water ${ }^{5,48,71}$ have shown that a simple Gaussian model is not adequate for a satisfactory description of the single particle proton dynamics. The model distribution function, both for the constant angle and the constant $q$ spectra, can be regarded as a nonGaussian function, expressed by a Gaussian lineshape multiplied by a truncated series of even Hermite polynomials:

$$
\begin{aligned}
F^{\exp }(y, \chi)= & \frac{\alpha}{\sqrt{2 \pi \sigma^{2}}} e^{-u^{2}}\left[1+c_{4} \frac{2}{3} H_{4}(u)\right. \\
& \left.+\ldots c_{3} \frac{1}{q} H_{3}(u)+\ldots\right] \otimes R_{n}(y, \chi),
\end{aligned}
$$

with $\chi$ being $2 \theta$ or $q$, and $u=\left(y-y_{0}\right) / \sqrt{2 \sigma^{2}}$. The $c_{n}$ are related to $a_{n}$ coefficients of Eq. (2.8) and, in particular, $c_{4}=3 / 64 a_{2}$. The fitting parameters are $\alpha$ (a normalization factor), $y_{0}, \sigma, c_{4}$, and $c_{3}$, the latter being the fourth and the third Hermite polynomials coefficients. It has to be stressed that for a truncated expansion of Eq. (4.1) up to the $H_{4}(u)$ term it turns out that the first non-Gaussian coefficient, $c_{4}$, is

$$
c_{4}=\frac{1}{64} \delta
$$

where $\delta$ is the kurtosis of the single particle momentum distribution, given by $\delta=\mu_{4}-3\left\langle\sigma^{2}\right\rangle^{2} /\left\langle\sigma^{2}\right\rangle^{2}, \mu_{4}$ being the fourth moment of the momentum distribution. ${ }^{72}$ This expression has been used for all the analysis performed: both at constant $q$ and constant angle and for both pores. Moreover, for each sample, two distinct set of fits have been performed using the same lineshape of Eq. (4.1): individual and simultaneous fits on the whole set of $F^{\exp }(y, q)$ and $F_{n}^{\exp }(y, 2 \theta)$ spectra. Table III reports results obtained from the fit on the individual $F_{n}^{\exp }(y, 2 \theta)$ spectra, for hydrated xerogel samples, at three selected scattering angles, for both pore sizes. A simultaneous fit of the whole individual spectra has also been performed, using a unique non-Gaussian model function [Eq. (4.1)] convoluted with the resolution functions corresponding to each scattering angle. Results for dry and hydrated samples, obtained from simultaneous fits on $F_{n}^{\exp }(y, 2 \theta)$ and $F^{\exp }(y, q)$, are shown in Tables IV and V, respectively. From the three tables one can note that the values of the physical quantities $\sigma,\left\langle E_{K}\right\rangle$, and $c_{4}$ are in good agreement within the statistical uncertainties. This feature demonstrates the internal consistency of the individual and simultaneous fitting procedures. In addition, the simultaneous fits reduce the uncertainties in the whole set of physical quantities, allowing a reliable lineshape analysis of the response function and momentum distribution, with special regard to non-Gaussian components, represented by the Hermite polynomial coefficients. The non-Gaussian coefficient $c_{4}$ is the only term which needs to be included in the whole fitting procedure in order to reproduce the experimental data with good statistical accuracy. This can be immediately appreciated from Fig. 4, where the $F_{n}^{\exp }(y, 2 \theta)$ function and the fitting results from corresponding simple Gaussian and non-Gaussian models are reported. For all samples, values of the $c_{3}$ parameter were

TABLE V. Results obtained from simultaneous fit of $F^{\exp }(y, q)$ data for both dry xerogel and hydrated xerogel samples, for pore sizes of 24 and $82 \AA$. The suffixes $D$ and $H$ refer to dry and hydrated xerogel samples: $\sigma_{D}, \sigma_{H}$ and $\left\langle E_{K}\right\rangle_{D},\left\langle E_{K}\right\rangle_{H}$ represent standard deviations and mean kinetic energies of dry and hydrated samples. The $c_{4 D}$ and $c_{4 H}$ are the non-Gaussian coefficients, resulting from the fits.

\begin{tabular}{cccccccc}
\hline \hline & \multicolumn{3}{c}{$\mathrm{Xerogel}_{D}$} & & \multicolumn{3}{c}{$\operatorname{Xerogel}_{H}$} \\
\cline { 2 - 3 } $\begin{array}{c}c \\
(\AA)\end{array}$ & $\begin{array}{c}\sigma_{D} \\
\left(\AA^{-1}\right)\end{array}$ & $\begin{array}{c}c_{4 D} \\
\left(\times 10^{-2}\right)\end{array}$ & $\begin{array}{c}\left\langle E_{K}\right\rangle_{D} \\
(\mathrm{meV})\end{array}$ & & $\begin{array}{c}\sigma_{H} \\
\left(\AA^{-1}\right)\end{array}$ & $\begin{array}{c}c_{4 H} \\
\left(\times 10^{-2}\right)\end{array}$ & $\begin{array}{c}\left\langle E_{K}\right\rangle_{H} \\
(\mathrm{meV})\end{array}$ \\
\hline 24 & $6.73 \pm 0.07$ & $2.98 \pm 0.12$ & $282 \pm 5$ & & $5.81 \pm 0.03$ & $2.90 \pm 0.06$ & $210 \pm 2$ \\
82 & $6.51 \pm 0.27$ & $2.63 \pm 0.47$ & $264 \pm 10$ & & $5.49 \pm 0.10$ & $1.53 \pm 0.21$ & $187 \pm 6$ \\
\hline \hline
\end{tabular}




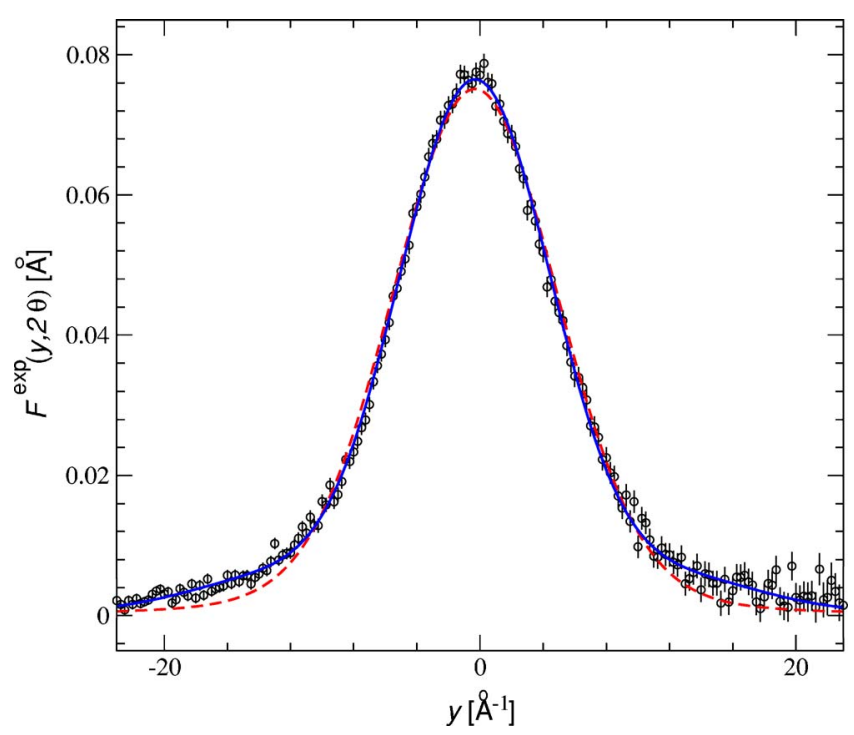

FIG. 4. (Color online) Experimental spectrum (circles) for water confined in xerogel with average pore diameter $d=24 \AA$ at scattering angle of $44.7^{\circ}$. The corresponding results of the fits using a Gaussian response function (dashed line) and a non-Gaussian response function (continuous line) are also shown.

zero within uncertainty; therefore it is suggested that final state effects affect mainly the recoil peak position at low scattering angles (see Fig. 2) and do not modify appreciably the lineshape; these effects are fully accounted for by the fitting parameter $y_{0}$.

The $\left\langle E_{K}\right\rangle_{\mathrm{H}}$ and $c_{4 \mathrm{H}}$ values for $24 \AA$ pore size are larger than for $82 \AA$ pore size. In the former case, a higher $c_{4 \mathrm{H}}$ value is an indication of a more pronounced non-Gaussian character, with higher kurtosis, of the momentum distribution.

As a final step, results from the lineshape analysis of hydrated and dry sample data sets have been combined, with the aim of deriving the response function associated with water protons only. One has to note that in DINS measurements, recoil peaks from different kinds of hydrogen appear at the same time of flight and $y$ range. Thus, within the incoherent approximation, one can express the response functions for hydrated samples in terms of hydrogen atoms belonging to water and to silanol groups. From Eq. (3.3) the response function, for each individual detector, may be expressed as a weighted sum of these two components, $F_{W}^{\exp }(y, 2 \theta)$ and $F_{D}^{\exp }(y, 2 \theta)$, respectively:

$$
F_{H}^{\exp }(y, 2 \theta)=c F_{D}^{\exp }(y, 2 \theta)+(1-c) F_{W}^{\exp }(y, 2 \theta),
$$

which corresponds to [see Eq. (3.3)]:

$$
J_{H}(y, 2 \theta)=c J_{D}(y, 2 \theta)+(1-c) J_{W}(y, 2 \theta),
$$

where the total proton momentum response function, $J_{\mathrm{H}}(y, 2 \theta)$, has a second moment given by

$$
\sigma_{H}^{2}=c \sigma_{D}^{2}+(1-c) \sigma_{W}^{2} .
$$

Subscripts $W$ and $D$ label hydrogen contributions associated with water and silanol groups; subscript $\mathrm{H}$ refers to all hydrogen atoms (water and silanol groups). The coefficient $c$ represents the fraction of silanol/total hydrogen atoms in the samples. For both hydrated xerogel samples, individual $F_{\mathrm{H}}^{\exp }(y, 2 \theta)$ functions have been fitted keeping $F_{D}^{\exp }(y, 2 \theta)$ lineshapes fixed (see Table IV) and with $c, \sigma_{W}$, and $c_{4 W}$, for $F_{W}^{\exp }(y, 2 \theta)$, as free parameters. This procedure allowed to effectively fit the hydrated sample with larger pore diameter $(82 \AA)$ and to derive its momentum distribution lineshape. The values for $\sigma_{W}$ and $\left\langle E_{K}\right\rangle_{W}$ resulting from this fit are reported in Table VI (left panel). Values for bulk water are also listed for comparison. In the case of the hydrated sample with smaller pores $(24 \AA)$, the fitting procedure did not allow to fit all parameters with sufficient accuracy. In this case, $c$ has been determined by combining information from the adsorbed water content and spectra intensities: (a) by comparison of theoretical densities of the samples and experimental weight/volume ratio and (b) from the relative intensities of the hydrogen recoil peak with respect to $\mathrm{Si}, \mathrm{Al}$, and $\mathrm{O}$ peaks. This approach provides values of $c=31 \%$ and $\sigma_{W}=5.48 \AA^{-1}$ for pore sizes of $24 \AA$ and $c=26 \%$ and $\sigma_{W}=5.01 \AA^{-1}$ for pore size of $82 \AA$ (see Table VI, right panel). It has to be noted that for higher pore diameter, values of $c$ and $\sigma_{W}$ agree with those derived from the fitting procedure. A plot of $\left\langle E_{K}\right\rangle_{W}$ values as a function of pore diameter is reported in Fig. 5, together with value obtained from previous experiments on bulk water. ${ }^{6,47,71}$

From Table VI and Fig. 5 one observes that the $\left\langle E_{K}\right\rangle_{W}$ values of water confined in xerogel pores are always higher than in bulk water. The $\left\langle E_{K}\right\rangle_{W}$ value is about $40 \mathrm{meV}$ higher than the bulk for the smaller pore diameter. It is also higher than the value for an isolated water molecule, ${ }^{47}$ which presumably has the strongest covalent bond and hence the broadest momentum distribution in the bond direction. It is possible that the additional kinetic energy is largely due to the molecules near the surface being strongly bonded to the pore surface. In the smaller pores the fraction of water molecules interacting closely with the confining matrix surface is larger. One can estimate about $12 \%$ and $35 \%$ of water molecules "on the first adsorption layer" in pore diameters of 82 and $24 \AA$, respectively. Moreover kinetic energies, $\left\langle E_{K}\right\rangle_{D}$,

TABLE VI. Values of $\sigma_{W}$ and $\left\langle E_{K}\right\rangle_{W}$ relative to protons from confined water molecules, obtained from Eq. (4.5)

\begin{tabular}{|c|c|c|c|c|c|c|}
\hline \multirow[b]{2}{*}{$\begin{array}{c}d \\
(\AA)\end{array}$} & \multicolumn{3}{|c|}{ From fit [see Eq. (4.3)] } & \multicolumn{3}{|c|}{ From Eq. (4.5) } \\
\hline & $\begin{array}{c}c \\
(\%)\end{array}$ & $\begin{array}{c}\sigma_{W} \\
\left(\AA^{-1}\right)\end{array}$ & $\begin{array}{c}\left\langle E_{K}\right\rangle_{W} \\
(\mathrm{meV})\end{array}$ & $\begin{array}{c}c \\
(\%)\end{array}$ & $\begin{array}{c}\sigma_{W} \\
\left(\AA^{-1}\right)\end{array}$ & $\begin{array}{c}\left\langle E_{K}\right\rangle_{W} \\
(\mathrm{meV})\end{array}$ \\
\hline 24 & $\cdots$ & $\cdots$ & $\cdots$ & $31 \pm 3$ & $5.48 \pm 0.06$ & $187 \pm 4$ \\
\hline 82 & $29 \pm 2$ & $4.94 \pm 0.04$ & $152 \pm 3$ & $26 \pm 3$ & $5.01 \pm 0.04$ & $156 \pm 3$ \\
\hline bulk & $\cdots$ & $4.87 \pm 0.05$ & $147 \pm 3$ & $\cdots$ & $4.87 \pm 0.05$ & $147 \pm 3$ \\
\hline
\end{tabular}
(right panel) and by a two lineshape fit as in Eq. (4.3) (left panel). Last row reports values obtained from previous DINS measurements in bulk water ${ }^{47}$. 


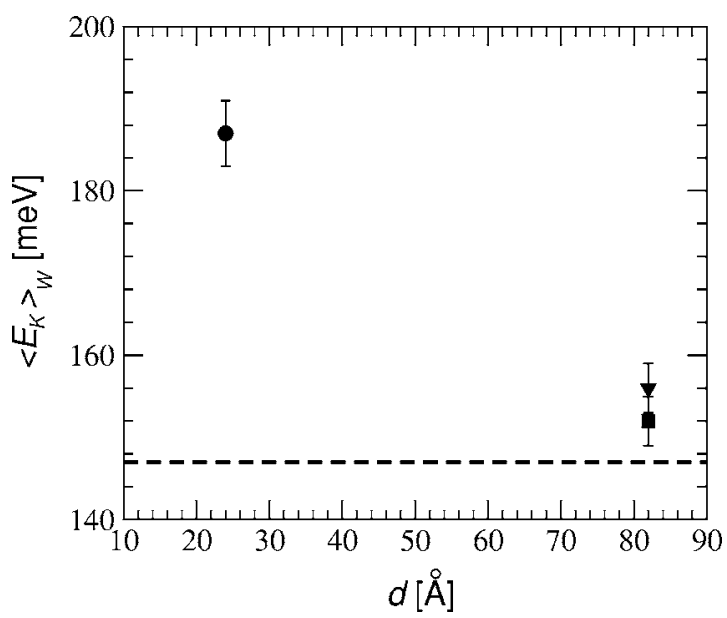

FIG. 5. Kinetic energies for protons in water confined in xerogel matrices, $\left\langle E_{K}\right\rangle_{W}$, as a function of the pore diameter. The dashed line represents value from previous experiments on bulk water (Ref. 47).

and momentum distributions $\left(\sigma_{D}, c_{4 D}\right.$ parameters $)$ of the dry samples are different for the two pore sizes, indicating a microscopic hydroxyl dynamics changing from 24 to $82 \AA$ diameter pores. Thus, the water proton dynamics of molecules close to the pore surface is modified by both geometrical effects of confinement and proximity to hydroxyl species of different microscopic dynamics. It is also possible that the increase in kinetic energy is due to the transverse motion of the protons in the bonds being confined more in the small pores than in bulk water.

It has to be noted that if $c, F_{D}^{\exp }(y, 2 \theta), F_{\mathrm{H}}^{\exp }(y, 2 \theta)$ are determined independently (see above), $F_{W}^{\exp }(y, 2 \theta)$ can be reconstructed by inverting Eq. (4.3):

$$
F_{W}^{\exp }(y, 2 \theta)=\frac{F_{H}^{\exp }(y, 2 \theta)-c F_{D}^{\exp }(y, 2 \theta)}{1-c} .
$$

$F_{W}^{\exp }(y, 2 \theta)$ has been thus determined using values of Table IV and $c$ values from Table VI, for both pore sizes.

Exploiting the relation between the response function and the momentum distribution [Eq. (2.5)], one can calculate the $n(p)$ for the protons in water confined in xerogel matrices. The resulting plots of spherical averages of the proton momentum distributions, $4 \pi p^{2} n(p)$, are shown in Fig. 6. Uncertainties in $n(p)$ functions, $\sqrt{\left\langle\delta n(p)^{2}\right\rangle}$ are expressed by the following expression; ${ }^{47}$

$$
\left\langle\delta n(p)^{2}\right\rangle=\sum_{i, j} \frac{\partial n(p)}{\partial \rho_{i}} \frac{\partial n(p)}{\partial \rho_{j}}\left\langle\delta \rho_{i} \delta \rho_{j}\right\rangle,
$$

where $\rho_{i}$ and $\rho_{j}$ stand for fit parameters, $\partial n(\mathbf{p}) / \partial \rho_{i}$ is the partial derivative of the momentum distribution relative to the $i$ th parameter, and $\left\langle\delta \rho_{i} \delta \rho_{j}\right\rangle$ is the element of the covariance matrix relative to $i$ th and $j$ th parameters.

From Fig. 6 it can be noted that $4 \pi p^{2} n(p)$ of confined water differs substantially from that of bulk liquid. In particular, it can be observed that lineshapes narrow in the range $10-15 \AA^{-1}$ and develop a second structure in the range $15-20 \AA^{-1}$. This behavior is more evident for the xerogel with $24 \AA$ pore diameter, with a minimum for $p=12 \AA^{-1}$, and a second maximum for $p=18 \AA^{-1}$. In this case the bimo-

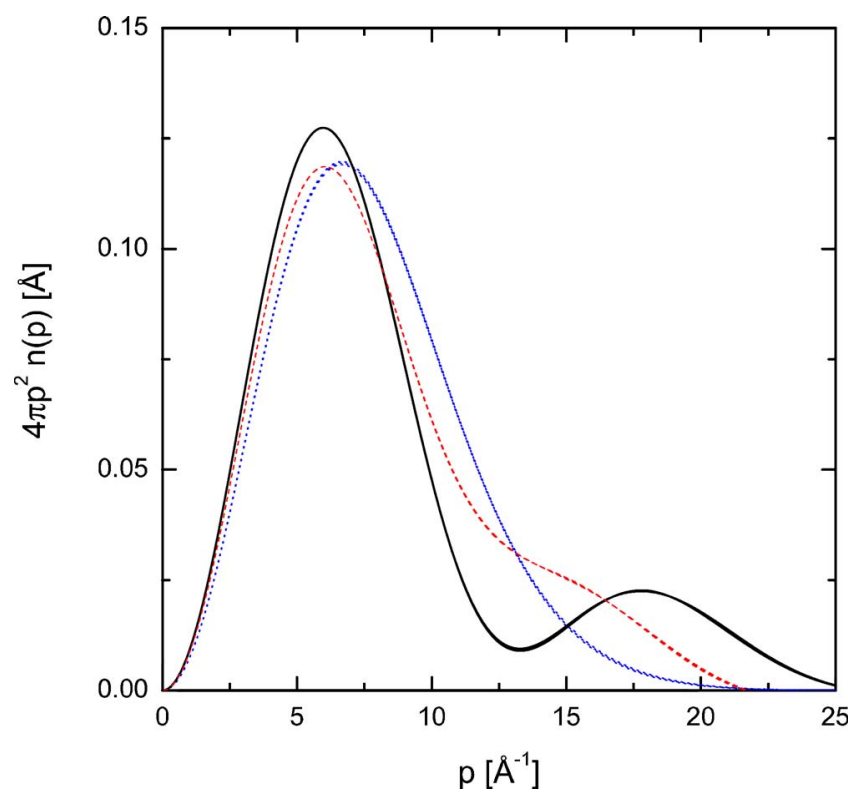

FIG. 6. (Color online) Spherical averages $4 \pi p^{2} n(p)$ and relative uncertainties $4 \pi p^{2} \sqrt{\left\langle\delta n(p)^{2}\right\rangle}$ (upper and lower lines) of the proton momentum distribution for water confined in xerogel matrices for pore diameters of $24 \AA$ (continuous line) and $82 \AA$ (dashed line), and for bulk water (dotted line) (from Ref. 47).

dal distribution suggests that changes in the local structure around protons occur, such as distortions of the hydrogen bond network. These are related to the local proton potential. We will infer the shape of this effective potential by fitting the momentum distribution with a model describing the motion along the bond by a wavefunction that corresponds in real space to two Gaussians separated by a distance $d .{ }^{6}$ When $d \neq 0$ one has an anisotropic momentum distribution given by

$$
n\left(p_{x}, p_{y}, p_{z}\right)=\frac{2 \cos ^{2}\left(p_{z} d / 2 \hbar\right)}{1+e^{-d^{2} \sigma_{z}^{2} / 2 \hbar^{2}}} \prod_{i} \frac{e^{-p_{i}^{2} / 2 \sigma_{i}^{2}}}{\left(2 \pi \sigma_{i}\right)^{1 / 2}} .
$$

This lineshape is spherically averaged and fitted using as fit parameters $\sigma_{z}, d$ and, for the transverse direction, $\sigma_{x}=\sigma_{y}{ }^{6}$ Figure 7 shows that a good fit of momentum distribution is obtained with values of fitting parameters given by $\sigma_{z}$ $=8.67 \AA^{-1}, \sigma_{x}=4.12 \AA^{-1}$, and $d=0.304 \AA$. The effective potential along the bond that gives rise to this momentum distribution and the ground state wavefunction itself are shown in Fig. 8, demonstrating clearly the bimodal nature of the ground state wavefunction that leads to the oscillation seen in Fig. 6.

The $n(p)$ for the water molecules in the smaller pores xerogel has been analyzed as if the molecules throughout the pore were in the same state. It is likely that the hydrogen bond network near the surface is more severely distorted than that in the interior. The success of the fit would seem to indicate that the molecules in the small pore are indeed in a very different state from that of bulk water and are strongly affected by confinement. In the larger pore, it would seem that a smaller fraction of strongly distorted molecules is present, consistent with the idea of a distorted surface layer. Further experiments with lower coverage of the pore surface 


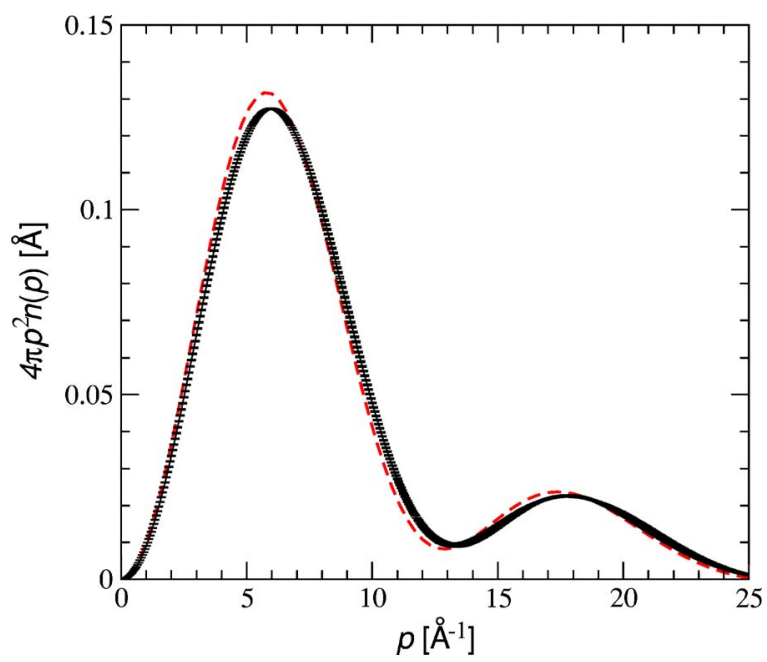

FIG. 7. (Color online) Proton radial momentum distribution $4 \pi p^{2} n(p)$ for water confined in xerogel matrices for pore diameter of $24 \AA$ obtained from the spherically averaged model function of Eq. (4.6) (dashed line) and from experimental data (continuous line with error bar).

would be needed to explore the nature of this surface layer. It is evident from Fig. 8 that this layer bears very little resemblance to bulk water. We note that coherence in another confined water system has been seen in water in carbon nanotubes at low temperatures ${ }^{73}$ and at the surface of proteins. ${ }^{74}$

\section{CONCLUSIONS}

In this paper, we have presented a DINS study on water adsorbed in silica xerogel matrices of different average pore sizes. These measurements allowed to determine the proton momentum distributions of adsorbed water molecules and the proton mean kinetic energy. A comparison with the value for bulk liquid water at ambient conditions is also possible. Results obtained for both fixed angle and constant $q$ spectra are in very good agreement with each other, for both the samples. The longitudinal momentum distribution is well described by a non-Gaussian lineshape, reflecting the anhar-

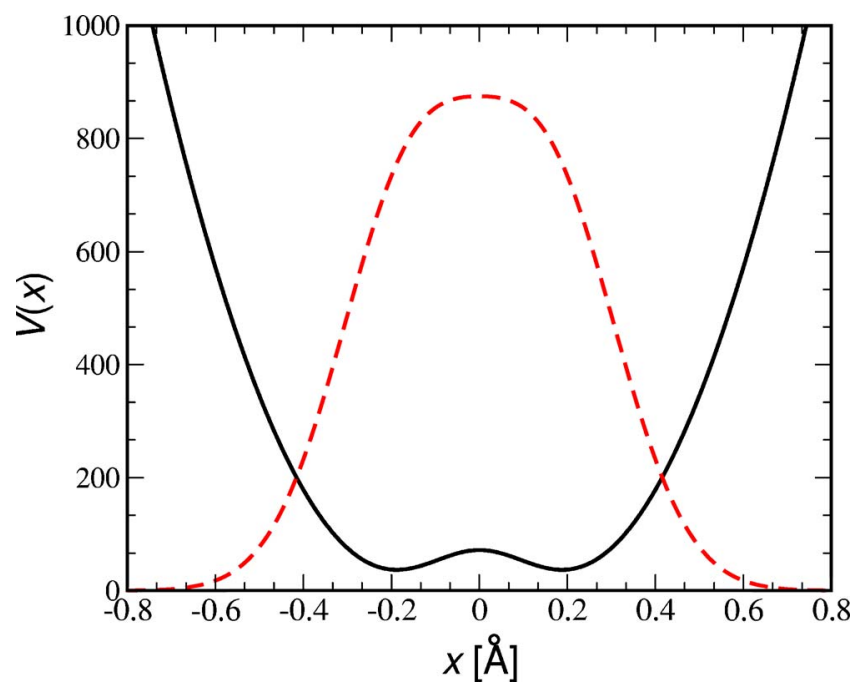

FIG. 8. (Color online) Effective single particle potential (continuous line) and wavefunction (dashed line) for protons in water confined in xerogel matrices for pore diameter of $24 \AA$. monic short-scale structure surrounding the protons. The non-Gaussian character of the momentum distribution is more pronounced for the smaller pores. The proton mean kinetic energy of water confined in xerogel is higher than the bulk water value. For the powder with average pore size of $82 \AA,\left\langle E_{K}\right\rangle_{W}$ is only few meV higher than for the bulk water while this value results sensibly higher for pore diameter of $24 \AA$. The layers of water near the surface of the pores are strongly perturbed, with the proton appearing to move coherently between two sites separated by $0.3 \AA$. The perturbation penetrates the entire pore in the smaller pore sample but is confined to a surface layer in the large pores. The changes of the water dynamics clearly depend on the interaction of water with the hydrophilic substrate. The nature of this interaction is not clear. The momentum distributions of the dry samples are different and yield different kinetic energies for the two pores, showing that the microscopic hydroxyl group dynamics are different in the two cases. The hydroxyl groups may play a role in the interaction, as might the morphology of the substrate. We want to mention also that molecular dynamics simulations of water confined in porous silica $^{18,41,42}$ have shown that both structure and dynamics reveal that water layers closest to the interface behave as they were at a lower temperature compared to that calculated from the simulation and are already approaching the glass transition at ambient conditions. Moreover the density profile throughout the pore diameter is not flat and shows a peak due to the strong hydrophilic character of the substrate surface at short distances from the confining wall, followed by a minimum. This modulation of the density may be responsible for the enhancement of the mean kinetic energy; at the same time evidence for quantum effects of the kind discussed in this paper may signal the vicinity to the glass transition. Whatever the origin of the interaction, the state of the water near the surfaces is qualitatively different form that of bulk water, and unlike any other form of water observed so far.

\section{ACKNOWLEDGMENTS}

Authors wish to thank D. Fernàndez Cañoto, F. Diré, and P. Prosposito for their assistance in the synthesis and characterization of xerogel samples. This work was supported within the CNR-CCLRC Agreement No. 01/9001 concerning collaboration in scientific research at the spallation neutron source ISIS. The financial support of the Consiglio Nazionale delle Ricerche in this research is hereby acknowledged. G.R. acknowledges the support of the DOE, Division of Basic Energy Sciences under Contract No. DE-FG0203ER46078.

\footnotetext{
${ }^{1}$ Hydration Processes in Biology: Theoretical and Experimental Approaches, NATO ASI Series A: Life Sciences, edited by M. C. Bellisent Funnel (IOS, Amsterdam, 1998), Vol. 305.

${ }^{2}$ O. Mishima and H. E. Stanley, Nature (London) 396, 329 (1998).

${ }^{3}$ A. K. Soper and M. A. Ricci, Phys. Rev. Lett. 84, 2881 (2000).

${ }^{4}$ P. G. Debenedetti, J. Phys.: Condens. Matter 15, R1669 (2003).

${ }^{5}$ C. Andreani, D. Colognesi, E. Degiorgi, and M. A. Ricci, J. Chem. Phys. 115, 11243 (2001).

${ }^{6}$ G. F. Reiter, J. C. Li, J. Mayers, T. Abdul-Redah, and P. Platzman, J. Phys. D 34, 142 (2004).

${ }^{7}$ V. Garbuio, M. Cascella, L. Reining, R. Del Sole, and O. Pulci, Phys. Rev. Lett. 97, 137402 (2006).
} 
${ }^{8}$ F. Mallamace, B. Broccio, C. Corsaro, A. Faraone, U. Wanderlingh, L. Liu, C. Y. Mou, and S. H. Chen, J. Phys. Chem. 124, 161102 (2006).

${ }^{9}$ P. G. Debenedetti, Metastable Liquids: Concepts and Principles (Princeton University Press, Princeton, 1997); G. W. Robinson, S. Singh, S.-B. Zhu, and M. W. Evans, Water in Biology, Chemistry and Physics (World Scientific, Singapore, 1999).

${ }^{10}$ W. Doster, A. Bachleitner, R. Dunau, M. Hiebl, and E. Luscher, Biophys. J. 50, 213 (1986).

${ }^{11}$ G. Sartor, A. Hallbrucker, K. Hofer, and E. Mayer, J. Phys. Chem. 96, 5133 (1992).

${ }^{12}$ K. Morishige and K. Nobuoka, J. Chem. Phys. 107, 6965 (1997); K. Morishige and K. Kawano, ibid. 110, 4867 (1999).

${ }^{13}$ F. Bruni, M. A. Ricci, and A. K. Soper, J. Chem. Phys. 109, 1478 (1998); A. K. Soper, F. Bruni, and M. A. Ricci, ibid. 109, 1486 (1998).

${ }^{14}$ S. H. Chen, P. Gallo, and M. C. Bellissent Funel, Can. J. Phys. 73, 703 (1995).

${ }^{15}$ J. M. Zanotti, M. C. Bellissent Funel, and S. H. Chen, Phys. Rev. E 59, 3084 (1998).

${ }^{16}$ L. D. Gelb, K. E. Gubbins, R. Radhakrishnan, and M. S. Bartkoviak, Rep. Prog. Phys. 61, 1573 (1999); M. C. Bellissent-Funel, S. Longeville, J. M. Zanotti, and S. H. Chen, Phys. Rev. Lett. 85, 3644 (2000).

${ }^{17}$ F. Venturini, P. Gallo, M. A. Ricci, A. R. Bizzarri, and S. Cannistraro, J. Chem. Phys. 114, 10010 (2001).

${ }^{18}$ P. Gallo, M. A. Ricci, and M. Rovere, J. Chem. Phys. 116, 342 (2002).

${ }^{19}$ R. Zangi, J. Phys.: Condens. Matter 16, S5371 (2004).

${ }^{20}$ S. H. Chen, L. Liu, E. Fratini, P. Baglioni, A. Faraone, and E. Mamontov, Proc. Natl. Acad. Sci. U.S.A. 103, 9012 (2006).

${ }^{21}$ V. Crupi, D. Majolino, P. Migliardo, and V. Venuti, J. Phys. Chem. B 106, 10884 (2002).

${ }^{22}$ S. Mitra, R. Mukhopadhyay, I. Tsukushi, and S. Ikeda, J. Phys.: Condens. Matter 13, 8455 (2001).

${ }^{23}$ E. Perelli-Cippo, C. Andreani, M. Casalboni, S. Dirè, D. FernandezCañoto, G. Gorini, S. Imberti, A. Pietropaolo, P. Prosposito, S. Schutzmann, R. Senesi, and M. Tardocchi, Physica B 385-386, 1095 (2006).

${ }^{24}$ C. F. Polzanek and R. G. Bryant, J. Chem. Phys. 81, 4038 (1984).

${ }^{25}$ A. Cupane, M. Levantino, and M. G. Santangelo, J. Phys. Chem. B 106, 11323 (2002).

${ }^{26}$ A. Scodinu and J. T. Fourkas, J. Phys. Chem. B 106, 10292 (2002).

${ }^{27}$ U. Raviv and J. Klein, Nature (London) 413, 51 (2001).

${ }^{28}$ U. Raviv, S. Giasson, J. Frey, and J. Klein, J. Phys.: Condens. Matter 14, 9275 (2002).

${ }^{29}$ H. E. Stanley and J. Teixeira, J. Chem. Phys. 73, 3404 (1980).

${ }^{30}$ M. C. Bellissent-Funel, R. Sridi Dorbez, and L. Bosio, J. Chem. Phys. 104, 10023 (1996).

${ }^{31}$ E. Spohr, C. Hartnig, P. Gallo, and M. Rovere, J. Mol. Liq. 80, 165 (1999)

${ }^{32}$ C. Hartnig, W. Witschel, E. Spohr, P. Gallo, M. A. Ricci, and M. Rovere, J. Mol. Liq. 85, 127 (2000).

${ }^{33}$ S. H. Lee, J. A. Mc Cammon, and P. J. Rossky, J. Chem. Phys. 80, 4448 (1984); S. H. Lee and P. J. Rossky, ibid. 100, 3334 (1994); J. X Fangetal, ibid. 107, 5212 (1997); S. B. Zhu and G. W. Robinson, ibid. 94, 1403 (1991).

${ }^{34}$ R. Lynden-Bell and J. C. Rasaiah, J. Chem. Phys. 105, 9266 (1996).

${ }^{35}$ J. Faeder and B. M. Ladanyi, J. Phys. Chem. B 104, 1033 (2000).

${ }^{36}$ I. Brovchenko, D. Paschek, and A. Geiger, J. Chem. Phys. 113, 5026 (2000).

${ }^{37}$ C. E. Capener and M. S. Sansom, J. Phys. Chem. B 106, 4543 (2002).

${ }^{38}$ O. Beckstein, P. C. Biggin, and M. S. Sansom, J. Phys. Chem. B 105 , 12902 (2001).

${ }^{39}$ P. Scheidler, W. Kob, and K. Binder, Europhys. Lett. 59, 701 (2002); F. W. Starr, T. B. Schroder, and S. C. Glotzer, Macromolecules 35, 4481 (2002).

${ }^{40}$ S. H. Chen and M. C. Bellissent-Funel, in Hydrogen Bond Networks,
NATO ASI Series C: Mathematical and Physical Sciences, edited by M. C. Bellissent-Funel and J. C. Dore (Kluwer Academic, Dordrecht, 1994), Vol. 435, p. 337.

${ }^{41}$ P. Gallo, M. Rovere, and E. Spohr, J. Chem. Phys. 113, 11324 (2000).

${ }^{42}$ P. Gallo, M. Rovere, and E. Spohr, Phys. Rev. Lett. 85, 4317 (2000).

${ }^{43}$ P. Gallo, Phys. Chem. Chem. Phys. 2, 1607 (2000).

${ }^{44}$ P. Gallo and M. Rovere, J. Phys.: Condens. Matter 15, 1521 (2002).

${ }^{45}$ M. Rovere and P. Gallo, Eur. Phys. J. E 12, 77 (2003).

${ }^{46}$ R. Senesi, C. Andreani, Z. Bowden, D. Colognesi, E. Degiorgi, A. L. Fielding, J. Mayers, M. Nardone, J. Norris, M. Praitano, N. J. Rhodes, W. G. Stirling, J. Tomkinson, and C. Uden, Physica B 276-278, S189 (2000).

${ }^{47}$ C. Andreani, D. Colognesi, J. Mayers, G. F. Reiter, and R. Senesi, Adv. Phys. 54, 377 (2005)

${ }^{48}$ C. Andreani, E. Degiorgi, R. Senesi, F. Cilloco, D. Colognesi, J. Mayers, M. Nardone, and E. Pace, J. Chem. Phys. 114, 387 (2001).

${ }^{49}$ V. F. Sears, Phys. Rev. B 30, 44 (1984).

${ }^{50}$ J. M. F. Gunn, C. Andreani, and J. Mayers, J. Phys. C 19, L835 (1986).

${ }^{51}$ C. Andreani, D. Colognesi, and E. Pace, Phys. Rev. B 60, 10008 (1999).

${ }^{52}$ G. B. West, Phys. Lett., C 18C, 263 (1975).

${ }^{53}$ G. F. Reiter and R. Silver, Phys. Rev. Lett. 54, 1047 (1985).

${ }^{54}$ C. J. Brinker, G. W. Scherer, Sol-Gel Science: The Physics and Chemistry of Sol-Gel Processing (Academic, San Diego, 1990).

${ }^{55}$ D. Fernandez-Cañoto, Ph.D. thesis, University of Vigo (Spain), University of Rome Tor Vergata (Italy), 2006.

${ }^{56}$ S. Brunauer, P. H. Emmett, and E. Teller, J. Am. Chem. Soc. 60, 309 (1938).

${ }^{57}$ C. G. Windsor, Pulsed Neutron Scattering (Taylor \& Francis, London, 1981).

${ }^{58}$ P. A. Seeger, A. D. Taylor, and R. M. Brugger, Nucl. Instrum. Methods Phys. Res. A 240, 98 (1985).

${ }^{59}$ C. Andreani, D. Colognesi, E. Degiorgi, A. Filabozzi, M. Nardone, and E. Pace, Nucl. Instrum. Methods Phys. Res. A 497, 535 (2003).

${ }^{60}$ C. Andreani, G. Baciocco, and J. Mayers, Nucl. Instrum. Methods Phys. Res. A 276, 297 (1989).

${ }^{61}$ C. Andreani, A. Filabozzi, E. Pace, and J. Mayers, Phys. Rev. B 54, 6255 (1996).

${ }^{62}$ J. Mayers, A. L. Fielding, and R. Senesi, Nucl. Instrum. Methods Phys. Res. A 481, 454 (2002).

${ }^{63}$ A. L. Fielding and J. Mayers, Nucl. Instrum. Methods Phys. Res. A 480, 680 (2002).

${ }^{64}$ V. V. Potapov and L. T. Zhuravlev, Glass Phys. Chem. 31, 661 (2005).

${ }^{65}$ S. Imberti, C. Andreani, V. Garbuio, G. Gorini, A. Pietropaolo, R. Senesi, and M. Tardocchi, Nucl. Instrum. Methods Phys. Res. A 552, 463 (2005).

${ }^{66}$ B. Dorner, Nucl. Instrum. Methods Phys. Res. B 247, 390 (2006), and references therein.

${ }^{67}$ K. H. Andersen, W. G. Stirling, R. Scherm, A. Stunault, B. Fak, H. Godfrin, and A. J. Dianoux, J. Phys.: Condens. Matter 6, 821 (1994)

${ }^{68}$ Momentum Distributions, edited by R. N. Silver and P. E. Sokol (Plenum, New York, 1989).

${ }^{69}$ R. Senesi, C. Andreani, and D. Colognesi, J. Low Temp. Phys. 126, 57 (2002).

${ }^{70}$ C. Andreani, C. Pantalei, and R. Senesi, Phys. Rev. B 75, 064515 (2007); J. Phys.: Condens. Matter 18, 5587 (2006).

${ }^{71}$ A. Pietropaolo, C. Andreani, A. Filabozzi, R. Senesi, G. Gorini, E. Perelli-Cippo, M. Tardocchi, N. J. Rhodes, and E. M. Schooneveld, Journal of Instrumentation 1, P04001 (2006).

${ }^{72}$ H. R. Glyde, Phys. Rev. B 50, 6726 (1996).

${ }^{73}$ G. Reiter, C. Burnham, D. Homouz, P. Platzman, J. Mayers, T. AbdulRedah, A. P. Moravsky, J. C. Li, C.-K. Loong, and A. Kolesnikov, Phys. Rev. Lett. 97, 247801 (2006).

${ }^{74}$ R. Senesi, A. Pietropaolo, A. Bocedi, S. E. Pagnotta, and F. Bruni, Phys. Rev. Lett. 98, 138102 (2007). 